Proceedings of the Edinburgh Mathematical Society (2005) 48, 691-704 (C)

DOI:10.1017/S0013091504000215 Printed in the United Kingdom

\title{
ANALYSIS OF AN EIGENVALUE PROBLEM ARISING IN A THERMAL WAVE PROPAGATION PROBLEM
}

\author{
D. J. NEEDHAM AND A. C. KING \\ Department of Mathematics, University of Reading, Whiteknights, \\ PO Box 220, Reading RG6 6AX, UK (d.j.needham@reading.ac.uk)
}

(Received 7 March 2004)

Abstract In this paper we study a non-standard eigenvalue problem which arises in the context of a thermal wave propagation problem, and some generalizations thereof. The eigenvalue distribution is fully explored and useful bounds on the location of the eigenvalues are obtained.

Keywords: eigenvalue problem; thermal wave propagation

2000 Mathematics subject classification: Primary 45C05

\section{Introduction}

There is a variety of industrial processes where a fuel, typically propane or butane, is burnt with the help of a catalyst. This usually results in a lower reaction temperature than is normal and will therefore produce less pollution (see $[\mathbf{2 , 3}]$, in which the modelling of the above processes is discussed in detail). If this takes place in a tube, with the catalyst coating the inner surface of the tube, then the governing equations for the gas flow and temperature $(\theta)$ can, in certain physical limits, be combined and cast into the form,

$$
\left[c+2\left(1-x^{2}\right)\right] \frac{\partial \theta}{\partial \xi}=\frac{1}{x} \frac{\partial}{\partial x}\left(x \frac{\partial \theta}{\partial x}\right)+\frac{1}{q^{2}} \frac{\partial^{2} \theta}{\partial \xi^{2}}
$$

for $0<x<1,-\infty<\xi<\infty$. The partial differentiation equation in which $c$ and $q$ are positive physical constants and $x$ and $\xi$ are coordinates along the radius and axis of the tube respectively, needs to be solved subject to the boundary conditions:

$$
\begin{aligned}
& \frac{\partial \theta}{\partial x}=0 \quad \text { on } x=0,-\infty<\xi<\infty ; \\
& \frac{\partial \theta}{\partial x}=0 \quad \text { on } x=1,-\infty<\xi<0 ; \\
& \frac{\partial \theta}{\partial x}=\mu(1-\theta) \quad \text { on } x=1,0<\xi<\infty ; \\
& \theta \rightarrow 0 \quad \text { as } \xi \rightarrow-\infty, \theta \rightarrow 1 \quad \text { as } \xi \rightarrow+\infty \text { for all } 0 \leqslant x \leqslant 1 .
\end{aligned}
$$


The solution to this linear elliptic boundary value problem is complicated by the split boundary conditions on $x=1$ and the variable coefficient in the $\theta_{\xi}$ term and the use of a Wiener-Hopf technique is hampered by this complication. If an eigenfunction expansion involving terms of the form $\phi(x, z)=\mathrm{e}^{\lambda \xi} \psi(x)$ is attempted in the domain $\xi<0$, then one is faced with attempting to solve

$$
\frac{1}{x} \frac{\mathrm{d}}{\mathrm{d} x}\left(x \frac{\mathrm{d} \psi}{\mathrm{d} x}\right)+\left[\frac{\lambda^{2}}{q^{2}}-\lambda\left(c+2\left(1-x^{2}\right)\right)\right] \psi=0, \quad 0<x<1,
$$

subject to $\psi_{x}(0)=\psi_{x}(1)=0$.

This eigenvalue problem, which is the subject of this paper, is linear in the function $\psi$ but nonlinear in the eigenvalue parameter $\lambda$ and therefore is of a non-standard form and has some interesting and unusual properties. These are investigated in both a rigorous analytic and asymptotic manner in the first part of this paper including $\S \S 2$ and 3 . The second, rather shorter, part of the paper describes an efficient numerical method to construct the eigenvalues and eigenfunctions and confirms the asymptotic results, which are presented in $\S 4$.

\section{The eigenvalue problem}

We now examine the eigenvalue problem,

$$
\begin{gathered}
{\left[x \psi^{\prime}\right]^{\prime}+\left[\frac{\lambda^{2}}{q^{2}}-\lambda G(x)\right] x \psi=0, \quad x \in(0,1),} \\
\psi(x), \psi^{\prime}(x) \text { bounded as } x \rightarrow 0 \\
\psi^{\prime}(1)=0
\end{gathered}
$$

with $\lambda$ being the eigenvalue parameter. Here $q>0$ is a constant, and $G:[0,1] \rightarrow \mathbb{R}$ is given by

$$
G(x)=2\left(1-x^{2}\right)+c \quad \forall x \in[0,1],
$$

with the constant $c>0$. We observe that

$$
G(x)>0 \quad \forall x \in[0,1] .
$$

We say that $\lambda=\lambda^{*} \in \mathbb{C}$ is an eigenvalue of (2.1)-(2.3) (which we henceforth refer to as $(\mathrm{E}))$ if there exists a non-trivial function $\psi=\psi^{*}:[0,1] \rightarrow \mathbb{C}$, with $\psi^{*} \in C[0,1] \cap$ $C^{2}(0,1) \cap C^{1}(0,1]$ such that $\psi=\psi^{*}$ solves (E) when $\lambda=\lambda^{*}$. We now examine the structure of the set of eigenvalues to $(\mathrm{E})$. We begin with the following proposition.

Proposition 2.1. The eigenvalues of $(\mathrm{E})$ are all real.

Proof. Let $\lambda^{*} \in \mathbb{C}$ be an eigenvalue of $(\mathrm{E})$ with eigenfunction, $\psi^{*}:[0,1] \rightarrow \mathbb{C}$. Then $\psi^{*}(x) \not \equiv 0$ on $[0,1]$. We normalize $\psi^{*}$ so that

$$
\int_{0}^{1} x \psi^{*}(x) \bar{\psi}^{*}(x) \mathrm{d} x=1
$$


Now from Equation (2.1) we obtain

$$
\left[x \psi^{* \prime} \bar{\psi}^{*}\right]_{0}^{1}-\int_{0}^{1} x \psi^{* \prime}\left(\bar{\psi}^{*}\right)^{\prime} \mathrm{d} x+\frac{\lambda^{* 2}}{q^{2}} \int_{0}^{1} x \psi^{*} \bar{\psi}^{*} \mathrm{~d} x-\lambda^{*} \int_{0}^{1} x G(x) \psi^{*} \bar{\psi}^{*} \mathrm{~d} x=0 .
$$

However, $\left(\bar{\psi}^{*}\right)^{\prime}=\overline{\left(\psi^{* \prime}\right)}$, and so, using conditions $(2.2),(2.3)$ and $(2.6)$, we find that $\lambda^{*}$ must be a root of the quadratic equation

$$
\Lambda^{2}+b \Lambda+c=0
$$

where

$$
b=-q^{2} \int_{0}^{1} x G(x)\left|\psi^{*}(x)\right|^{2} \mathrm{~d} x<0 \quad \text { and } \quad c=-q^{2} \int_{0}^{1} x\left|\psi^{*}(x)^{\prime}\right|^{2} \mathrm{~d} x<0
$$

Now using (2.9) we have

$$
b^{2}-4 c=q^{4}\left\{\int_{0}^{1} x G(x)\left|\psi^{*}(x)\right|^{2} \mathrm{~d} x\right\}^{2}+4 q^{2} \int_{0}^{1} x\left|\psi^{*}(x)^{\prime}\right|^{2} \mathrm{~d} x>0,
$$

and so any root $\Lambda \in \mathbb{C}$ of (2.8) must be real, and hence $\lambda^{*} \in \mathbb{R}$, as required.

Thus, we may now restrict attention to $(\mathrm{E})$ with $\lambda \in \mathbb{R}$. To begin with, we consider the modified problem:

$$
\begin{gathered}
{\left[x \phi^{\prime}\right]^{\prime}+[\gamma-\lambda G(x)] x \phi=0, \quad x \in(0,1),} \\
\phi(x), \phi^{\prime}(x) \text { bounded as } x \rightarrow 0, \\
\phi^{\prime}(1)=0,
\end{gathered}
$$

which we will refer to as $\mathrm{P}[\lambda]$. Here, $\gamma \in \mathbb{C}, \lambda \in \mathbb{R}$, and for each fixed $\lambda \in \mathbb{R}$, we will regard $\mathrm{P}[\lambda]$ as an eigenvalue problem with eigenvalue parameter $\gamma \in \mathbb{C}$. An examination of $\mathrm{P}[\lambda]$ shows that for each fixed $\lambda \in \mathbb{R}$, then $\mathrm{P}[\lambda]$ is a singular Sturm-Louiville eigenvalue problem, with eigenvalue parameter $\gamma \in \mathbb{C}$. In particular, for any $\gamma \in \mathbb{C}$, let $\phi_{+}(x)$ and $\phi_{-}(x)$ be two linearly independent solutions of Equation $(2.10)$ on $[0,1]$. Then $\phi_{+}(x)$ may be chosen so that it is analytic at $x=0$, while $\phi_{-}(x)$ is singular at $x=0$. Specifically, $\phi_{+}(x)$ may be chosen so that

$$
\phi_{+}(x)=1+\frac{1}{4}\left[\lambda(2-c)-\frac{\lambda^{2}}{q}\right] x^{2}+O\left(x^{3}\right) \quad \text { as } x \rightarrow 0^{+},
$$

while $\phi_{-}(x)$ has

$$
\phi_{-}(x)=\log x+O\left(x^{2} \log x\right) \quad \text { as } x \rightarrow 0^{+} .
$$

The principle solution of Equation (2.10) is thus $\phi_{+}(x)$, and it is readily verified that the singular Sturm-Louiville problem $\mathrm{P}[\lambda]$ is in the limit circle non-oscillatory class. Thus, the classical Sturm-Louiville theory (see, for example, $[\mathbf{1}$, Chapter 8$]$ ) applies to $\mathrm{P}[\lambda]$ for each fixed $\lambda \in \mathbb{R}$, which establishes the following statements for $\mathrm{P}[\lambda]$. 
(i) All of the eigenvalues of $\mathrm{P}[\lambda]$ are real, and form a countably infinite set, say, $\gamma_{0}(\lambda), \gamma_{1}(\lambda), \ldots$, with

$$
-\infty<\gamma_{0}(\lambda)<\gamma_{1}(\lambda)<\gamma_{2}(\lambda)<\cdots
$$

and

$$
\gamma_{n}(\lambda) \rightarrow \infty \quad \text { as } n \rightarrow \infty
$$

(ii) Each eigenvalue $\gamma=\gamma_{n}(\lambda)$ has a one-dimensional eigenspace with normalized eigenfunction $\phi_{n}:[0,1] \rightarrow \mathbb{R}$, such that

$$
\int_{0}^{1} x \phi_{n}(x, \lambda)^{2} \mathrm{~d} x=1
$$

(iii) Distinct eigenvalues $\gamma=\gamma_{n}(\lambda)$ and $\gamma=\gamma_{m}(\lambda)$ have orthogonal eigenfunctions, that is, for $n \neq m$,

$$
\int_{0}^{1} x \phi_{n}(x, \lambda) \phi_{m}(x, \lambda) \mathrm{d} x=0
$$

Now, for each fixed $n=0,1,2, \ldots$, we may regard $\gamma=\gamma_{n}(\lambda)$ as a function of $\lambda \in \mathbb{R}$, and it follows from the analytic dependence of Equation (2.10) on $\lambda$ and $\gamma$ that $\gamma_{n}: \mathbb{R} \rightarrow \mathbb{R}$ is continuous and has continuous derivative, that is $\gamma_{n} \in C^{1}(\mathbb{R})$. Moreover, via (i), the curves $\gamma=\gamma_{n}(\lambda)$ are non-intersecting in the $(\lambda, \gamma)$-plane. In addition, it is also straightforward to establish, via Froebenius theory, that for each $n=0,1,2, \ldots$, then $\phi_{n}(x, \lambda)$ is such that $\phi_{n} \in C^{3}([0,1] \times \mathbb{R})$, and, moreover,

$$
\phi_{n x}(0, \lambda)=0 \quad \forall \lambda \in \mathbb{R} .
$$

Now, for each fixed $n=0,1,2, \ldots$, we examine the properties of the corresponding function $\gamma=\gamma_{n}(\lambda)$ in more detail. For $\gamma=\gamma_{n}(\lambda)$, the corresponding eigenfunction $\phi=\phi_{n}(x, \lambda)$ then satisfies

$$
\begin{aligned}
{\left[x \phi_{n x}\right]_{x}+\left[\gamma_{n}(\lambda)-\lambda G(x)\right] x \phi_{n} } & =0, \quad x \in(0,1), \\
\phi_{n x}(0, \lambda)=\phi_{n x}(1, \lambda) & =0 .
\end{aligned}
$$

Since $\phi_{n} \in C^{3}([0,1] \times \mathbb{R})$ and $\gamma_{n} \in C^{1}[\mathbb{R}]$, we can differentiate through both (2.14) and (2.15) with respect to $\lambda$ to obtain

$$
\begin{aligned}
{\left[x \chi_{n x}\right]_{x}+\left[\gamma_{n}(\lambda)-\lambda G(x)\right] x \chi_{n} } & =-\left[\gamma_{n}^{\prime}(\lambda)-G(x)\right] x \phi_{n}, \quad x \in(0,1), \\
\chi_{n x}(0, \lambda) & =\chi_{n x}(1, \lambda)=0
\end{aligned}
$$

where $\chi_{n}(x, \lambda)=\phi_{n \lambda}(x, \lambda)$ for all $(x, \lambda) \in[0,1] \times \mathbb{R}$. Therefore, $\chi_{n}(x, \lambda)$ provides a solution to (2.16), (2.17) for any $\lambda \in \mathbb{R}$. However, we can regard (2.16), (2.17) as an inhomogeneous boundary value problem for $\chi_{n}(x, \lambda)$ on $x \in[0,1]$, and since $\phi_{n}(x, \lambda)$ is 
a non-trivial solution of the homogeneous part, and $\chi_{n}(x, \lambda)=\phi_{n \lambda}(x, \lambda)$ solves $(2.16)$, (2.17), then the Fredholm theory (see, for example, [4]) demands that

$$
\int_{0}^{1}\left[\gamma_{n}^{\prime}(\lambda)-G(x)\right] x \phi_{n}^{2}(x, \lambda) \mathrm{d} x=0
$$

and so, using (ii) in (2.18), we must have

$$
\gamma_{n}^{\prime}(\lambda)=\int_{0}^{1} G(x) x \phi_{n}^{2}(x, \lambda) \mathrm{d} x>0
$$

for each $\lambda \in \mathbb{R}$ and $n=0,1,2, \ldots$ Thus we have the following proposition.

Proposition 2.2. The functions $\gamma_{n}: \mathbb{R} \rightarrow \mathbb{R}$, for each $n=0,1,2, \ldots$, are such that $\gamma_{n}(\lambda)$ are strictly monotone increasing with $\lambda \in \mathbb{R}$, and satisfy the inequalities

$$
c \lambda+\mu_{n}^{2} \leqslant \gamma_{n}(\lambda) \leqslant(2+c) \lambda+\mu_{n}^{2} \text { for all } \lambda \geqslant 0,
$$

while

$$
(2+c) \lambda+\mu_{n}^{2} \leqslant \gamma_{n}(\lambda) \leqslant c \lambda+\mu_{n}^{2} \quad \text { for all } \lambda \leqslant 0
$$

Moreover,

$$
\gamma_{n}(0)=\mu_{n}^{2} \quad \text { for } n=0,1,2, \ldots
$$

where

$$
0=\mu_{0}<\mu_{1}<\mu_{2}<\cdots
$$

are the non-negative roots of the equation $J_{1}(X)=0$, with $J_{1}(X)$ being the Bessel function of order one.

Proof. For fixed $n=0,1,2, \ldots$, strict monotonicity of $\gamma_{n}(\lambda)$ with $\lambda \in \mathbb{R}$ follows from (2.19). We next observe that

$$
c \leqslant G(x) \leqslant 2+c \text { for all } x \in[0,1] .
$$

Thus, it follows from (ii) and (2.19) that

$$
c \leqslant \gamma_{n}^{\prime}(\lambda) \leqslant 2+c \text { for all } \lambda \in \mathbb{R}
$$

Application of $\int_{0}^{\lambda} \cdots \mathrm{d} s$ to (2.21) establishes that, for $\lambda \geqslant 0$,

$$
c \lambda+\gamma_{n}(0) \leqslant \gamma_{n}(\lambda) \leqslant(2+c) \lambda+\gamma_{n}(0) .
$$

Similarly, application of $\int_{\lambda}^{0} \cdots \mathrm{d} s$ to $(2.21)$ establishes that, for $\lambda \leqslant 0$,

$$
(2+c) \lambda+\gamma_{n}(0) \leqslant \gamma_{n}(\lambda) \leqslant c \lambda+\gamma_{n}(0) .
$$

Now, when $\lambda=0, \mathrm{P}[0]$ is

$$
\begin{gathered}
{\left[x \phi^{\prime}\right]^{\prime}+\gamma x \phi=0, \quad x \in(0,1),} \\
\phi(x), \phi^{\prime}(x) \text { bounded as } x \rightarrow 0, \\
\phi^{\prime}(1)=0,
\end{gathered}
$$


and it is readily established that the eigenvalues of $\mathrm{P}[0]$ are given by $\gamma=\mu_{n}^{2}, n=$ $0,1,2, \ldots$, where

$$
0=\mu_{0}^{2}<\mu_{1}^{2}<\mu_{2}^{2}<\mu_{3}^{2}<\cdots
$$

and $\mu_{n}, n=0,1,2, \ldots$, are the non-negative zeros of the Bessel function $J_{1}(\mu)$. Hence, we conclude that

$$
\gamma_{n}(0)=\mu_{n}^{2}, \quad n=0,1,2, \ldots,
$$

and substitution into (2.22) and (2.23) completes the proof.

Proposition 2.3. For each $n=0,1,2, \ldots$, the function $\gamma_{n}: \mathbb{R} \rightarrow \mathbb{R}$ has a unique zero (which is simple) at, say, $\lambda=\sigma_{n}$, with

$$
0=\sigma_{0}>\sigma_{1}>\sigma_{2}>\cdots
$$

and $\sigma_{n} \rightarrow-\infty$ as $n \rightarrow \infty$.

Proof. Fix $n=0,1,2, \ldots$, then it follows from Proposition 2.2 that $\gamma_{n}(\lambda)$ is strictly monotone increasing with $\lambda \in \mathbb{R}$, and that

$$
\gamma_{n}(\lambda) \rightarrow \begin{cases}+\infty & \text { as } \lambda \rightarrow+\infty \\ -\infty & \text { as } \lambda \rightarrow-\infty\end{cases}
$$

We conclude that $\gamma_{n}(\lambda)$ has a unique (simple, since $\gamma_{n}^{\prime}(\lambda)>0$ ) zero at, say, $\lambda=\sigma_{n}$. It also follows from the inequalities in Proposition 2.2 that $\gamma_{0}(0)=0$ and $\gamma_{n}(0)>0$ for $n=1,2, \ldots$, and so $\sigma_{0}=0$ and $\sigma_{n}<0$ for all $n=1,2, \ldots$ Moreover, the ordering in (i) requires that

$$
0=\sigma_{0}>\sigma_{1}>\sigma_{2}>\sigma_{3}>\cdots
$$

In addition, the inequalities in Proposition 2.2 give

$$
-\frac{\mu_{n}^{2}}{c} \leqslant \sigma_{n} \leqslant-\frac{\mu_{n}^{2}}{2+c} \quad \text { for each } n=0,1,2, \ldots,
$$

and so

$$
\sigma_{n} \rightarrow-\infty \quad \text { as } n \rightarrow \infty
$$

The proof is complete.

We also have the following proposition.

Proposition 2.4. For each $n=0,1,2, \ldots$, the function $\gamma_{n}: \mathbb{R} \rightarrow \mathbb{R}$ is such that

$$
\gamma_{n}(\lambda) \rightarrow \begin{cases}+\infty & \text { as } \lambda \rightarrow+\infty \\ -\infty & \text { as } \lambda \rightarrow-\infty\end{cases}
$$

and

$$
\gamma_{n}(\lambda)=O(\lambda) \text { as }|\lambda| \rightarrow \infty .
$$

Proof. This follows directly from the inequalities in Proposition 2.2. 


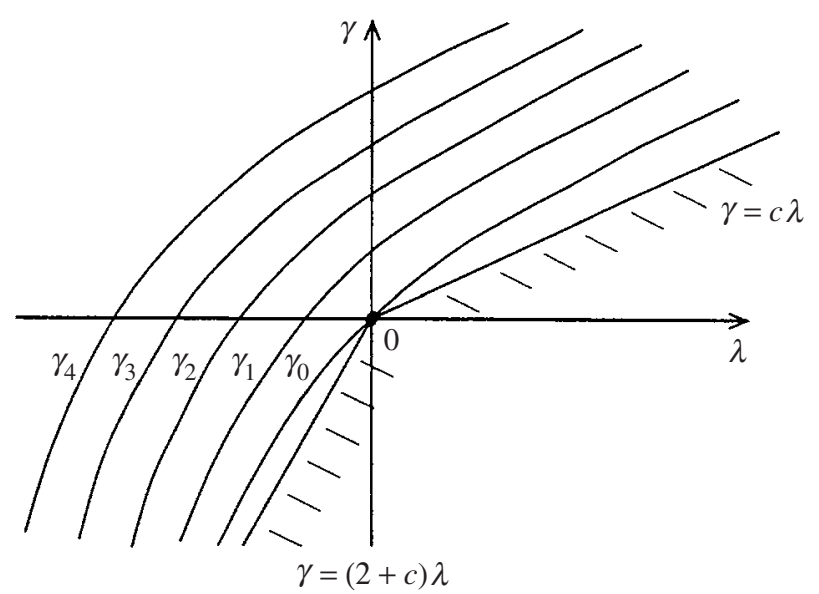

Figure 1. The functions $\gamma_{n}(\lambda), n=0,1,2, \ldots$, in the $(\lambda, \gamma)$-plane.

We can now sketch the functions $\gamma_{n}(\lambda)$ for each $n=0,1,2, \ldots$, and this is illustrated in Figure 1.

We now state the following theorem.

Theorem 2.5. The two-parameter eigenvalue problem (2.10)-(2.12) has a non-trivial solution if and only if

$$
(\lambda, \gamma) \in \bigcup_{n=0}^{\infty} \Lambda_{n}
$$

where $\Lambda_{n}=\left\{(\lambda, \gamma) \in \mathbb{R}^{2}: \gamma=\gamma_{n}(\lambda)\right\}$.

\section{Proof.}

$(\Leftarrow)$ Suppose that $\left(\lambda^{*}, \gamma^{*}\right) \in \bigcup_{n=0}^{\infty} \Lambda_{n}$, then $\gamma^{*}=\gamma_{n}\left(\lambda^{*}\right)$ for some $n=0,1,2, \ldots$ However, $\gamma_{n}\left(\lambda^{*}\right)$ is an eigenvalue of $\mathrm{P}\left[\lambda^{*}\right]$, and so $(2.10)-(2.12)$ has a non-trivial solution when $(\lambda, \gamma)=\left(\lambda^{*}, \gamma^{*}\right)$, as required.

$(\Rightarrow)$ Suppose for $(\lambda, \gamma)=\left(\lambda^{*}, \gamma^{*}\right)$ that $(2.10)-(2.12)$ has a non-trivial solution. Then $\gamma^{*}$ must be an eigenvalue of $\mathrm{P}\left[\lambda^{*}\right]$, and so $\gamma^{*}=\gamma_{n}\left(\lambda^{*}\right)$ for some $n=0,1,2, \ldots$, which requires $\left(\lambda^{*}, \gamma^{*}\right) \in \Lambda_{n}$ for some $n=0,1,2, \ldots$ and hence $\left(\lambda^{*}, \gamma^{*}\right) \in \bigcup_{n=0}^{\infty} \Lambda_{n}$.

Remark 2.6. For each $(\lambda, \gamma) \in \bigcup_{n=0}^{\infty} \Lambda_{n},(2.10)-(2.12)$ has a one-dimensional family of non-trivial solutions, which, for $(\lambda, \gamma) \in \Lambda_{n}(n=0,1,2, \ldots)$, is spanned by $\psi=$ $\phi_{n}(x, \lambda):[0,1] \rightarrow \mathbb{R}$.

We are now in a position to locate the eigenvalues of $(\mathrm{E})$. We first require three preliminary results.

Lemma 2.7. $\lambda^{*} \in \mathbb{R}$ is an eigenvalue of (E) if and only if $\left(\lambda^{*}, \lambda^{* 2} / q^{2}\right) \in \bigcup_{n=0}^{\infty} \Lambda_{n}$. 


\section{Proof.}

$(\Rightarrow)$ Suppose $\lambda^{*} \in \mathbb{R}$ is an eigenvalue of $(\mathrm{E})$, then there exists a non-trivial solution $\psi^{*}:[0,1] \rightarrow \mathbb{C}$ of $(2.1)-(2.3)$ when $\lambda=\lambda^{*}$. However, $\phi=\psi^{*}$ provides a non-trivial solution to $(2.10)-(2.12)$ when $(\lambda, \gamma)=\left(\lambda^{*}, \lambda^{* 2} / q^{2}\right)$, and so, via Theorem 2.5, $\left(\lambda^{*}, \lambda^{* 2} / q^{2}\right) \in \bigcup_{n=0}^{\infty} \Lambda_{n}$, as required.

$(\Leftarrow)$ Suppose $\left(\lambda^{*}, \lambda^{* 2} / q^{2}\right) \in \bigcup_{n=0}^{\infty} \Lambda_{n}$, then there exists a non-trivial solution $\phi^{*}:[0,1] \rightarrow$ $\mathbb{C}$ of (2.10)-(2.12), when $\lambda=\lambda^{*}$ and $\gamma=\lambda^{* 2} / q^{2}$, after which it is readily verified that $\psi=\phi^{*}$ provides a non-trivial solution to (2.1)-(2.3) when $\lambda=\lambda^{*}$. Hence, $\lambda=\lambda^{*}$ is an eigenvalue of $(\mathrm{E})$, as required.

Lemma 2.8. For each $n=0,1,2, \ldots$, the function $f_{n}: \mathbb{R} \rightarrow \mathbb{R}$, given by

$$
f_{n}(\lambda)=\gamma_{n}(\lambda)-\frac{\lambda^{2}}{q^{2}}, \quad \lambda \in \mathbb{R},
$$

has exactly two zeros, say, $\lambda=\lambda_{n}^{+}$and $\lambda=\lambda_{n}^{-}$, with

$$
0<\lambda_{0}^{+}<\lambda_{1}^{+}<\lambda_{2}^{+}<\cdots
$$

and

$$
0=\lambda_{0}^{-}>\lambda_{1}^{-}>\lambda_{2}^{-}>\cdots
$$

Moreover,

$$
\lambda_{n}^{+} \rightarrow+\infty \quad \text { and } \quad \lambda_{n}^{-} \rightarrow-\infty \quad \text { as } n \rightarrow \infty
$$

while

$$
\frac{q^{2} c+\left[q^{4} c^{2}+4 q^{2} \mu_{n}^{2}\right]^{1 / 2}}{2} \leqslant \lambda_{n}^{+} \leqslant \frac{q^{2}(2+c)+\left[q^{4}(2+c)^{2}+4 q^{2} \mu_{n}^{2}\right]^{1 / 2}}{2}
$$

and

$$
\frac{q^{2}(2+c)-\left[q^{4}(2+c)^{2}+4 q^{2} \mu_{n}^{2}\right]^{1 / 2}}{2} \leqslant \lambda_{n}^{-} \leqslant \frac{q^{2} c-\left[q^{4} c^{2}+4 q^{2} \mu_{n}^{2}\right]^{1 / 2}}{2}
$$

for each $n=0,1,2, \ldots$.

Proof. For $n=0,1,2, \ldots$, consider $f_{n}: \mathbb{R} \rightarrow \mathbb{R}$, given by

$$
f_{n}(\lambda)=\gamma_{n}(\lambda)-\frac{\lambda^{2}}{q^{2}}, \quad \lambda \in \mathbb{R} .
$$

It follows directly from Propositions $2.2-2.4$ that $f_{n}(\lambda)$ has exactly two zeros, at, say, $\lambda=\lambda_{n}^{+}$and $\lambda=\lambda_{n}^{-}$, with $\lambda_{0}^{-}=0$ and $\lambda_{0}^{+}>0$, while $\lambda_{n}^{-}<0$ and $\lambda_{n}^{+}>0$ for $n=1,2, \ldots$ Moreover, the orderings in (i) require that

$$
\cdots<\lambda_{2}^{-}<\lambda_{1}^{-}<\lambda_{0}^{-}=0<\lambda_{0}^{+}<\lambda_{1}^{+}<\lambda_{2}^{+} \cdots .
$$

In addition, the inequalities (2.24) and (2.25) are direct consequences of the inequalities in Proposition 2.2, while (2.24) and (2.25) establish that $\lambda_{n}^{+} \rightarrow+\infty$ and $\lambda_{n}^{-} \rightarrow-\infty$ as $n \rightarrow \infty$. 
Remark 2.9. We observe from (2.24) and (2.25) that

$$
\begin{array}{ll}
\lambda_{n}^{+} \sim q \mu_{n} & \text { as } n \rightarrow \infty, \\
\lambda_{n}^{-} \sim-q \mu_{n} & \text { as } n \rightarrow-\infty .
\end{array}
$$

Lemma 2.10. Let $A=\left\{(\lambda, \gamma) \in \mathbb{R}^{2}: \gamma=\lambda^{2} / q^{2}\right\}$. Then

$$
A \cap\left[\bigcup_{n=0}^{\infty} \Lambda_{n}\right]=\left\{\left(\lambda_{n}^{+}, \lambda_{n}^{+2} / q^{2}\right): n=0,1,2, \ldots\right\} \cup\left\{\left(\lambda_{n}^{-}, \lambda_{n}^{-2} / q^{2}\right): n=0,1,2, \ldots\right\} .
$$

Proof. Let $\left(\lambda^{*}, \gamma^{*}\right) \in A \cap\left[\bigcup_{n=0}^{\infty} \Lambda_{n}\right]$. Then $\gamma^{*}=\lambda^{* 2} / q^{2}$ and $\gamma^{*}=\gamma_{r}\left(\lambda^{*}\right)$ for some $r=0,1,2, \ldots$. Hence,

$$
\frac{\lambda^{* 2}}{q^{2}}=\gamma_{r}\left(\lambda^{*}\right)
$$

for some $r=0,1,2, \ldots$, and so $f_{r}\left(\lambda^{*}\right)=0$. Thus $\lambda^{*}=\lambda_{r}^{+}$or $\lambda_{r}^{-}$, so that $\gamma^{*}=\lambda_{r}^{+2} / q^{2}$ or $\lambda_{r}^{-2} / q^{2}$ accordingly, and we conclude that

$$
\left(\lambda^{*}, \gamma^{*}\right) \in\left\{\left(\lambda_{n}^{+}, \lambda_{n}^{+2} / q^{2}\right): n=0,1,2, \ldots\right\} \cup\left\{\left(\lambda_{n}^{-}, \lambda_{n}^{-2} / q^{2}\right): n=0,1,2, \ldots\right\}
$$

and so

$$
A \cap\left[\bigcup_{n=0}^{\infty} \Lambda_{n}\right] \subseteq\left\{\left(\lambda_{n}^{+}, \lambda_{n}^{+2} / q^{2}\right): n=0,1,2, \ldots\right\} \cup\left\{\left(\lambda_{n}^{-}, \lambda_{n}^{-2} / q^{2}\right): n=0,1,2, \ldots\right\} .
$$

Now consider $\left(\lambda_{r}^{+}, \lambda_{r}^{+2} / q^{2}\right)$ for any $r=0,1,2, \ldots$ Then clearly $\left(\lambda_{r}^{+}, \lambda_{r}^{+2} / q^{2}\right) \in A$. Also $\lambda_{r}^{+2} / q^{2}=\gamma_{r}\left(\lambda_{r}^{+}\right)$, by definition, so that $\left(\lambda_{r}^{+}, \lambda_{r}^{+2} / q^{2}\right) \in \Lambda_{r}$. Hence,

$$
\left(\lambda_{r}^{+}, \lambda_{r}^{+2} / q^{2}\right) \in A \cap\left[\bigcup_{n=0}^{\infty} \Lambda_{n}\right]
$$

A similar conclusion follows if we start with $\left(\lambda_{r}^{-}, \lambda_{r}^{-2} / q^{2}\right)$ for any $r=0,1,2, \ldots$ We conclude that

$$
\left\{\left(\lambda_{n}^{+}, \lambda_{n}^{+2} / q^{2}\right): n=0,1,2, \ldots\right\} \cup\left\{\left(\lambda_{n}^{-}, \lambda_{n}^{-2} / q^{2}\right): n=0,1,2, \ldots\right\} \subseteq A \cap\left[\bigcup_{n=0}^{\infty} \Lambda_{n}\right] .
$$

The result follows immediately from (2.26) and (2.27).

We now have the following theorem.

Theorem 2.11. The set of eigenvalues of (E) is given by

$$
\mathcal{E}=\left\{\lambda \in \mathbb{R}: \lambda=\lambda_{n}^{+}, n=0,1,2, \ldots\right\} \cup\left\{\lambda \in \mathbb{R}: \lambda=\lambda_{n}^{-}, n=0,1,2, \ldots\right\} .
$$

\section{Proof.}

$(\Rightarrow)$ Let $\lambda^{*}$ be an eigenvalue of $(\mathrm{E})$, then $\left(\lambda^{*}, \lambda^{* 2} / q^{2}\right) \in \bigcup_{n=0}^{\infty} \Lambda_{n}$, via Lemma 2.7. Hence, $\left(\lambda^{*}, \lambda^{* 2} / q^{2}\right) \in A \cap\left[\bigcup_{n=0}^{\infty} \Lambda_{n}\right]$, and so, via Lemma $2.10, \lambda^{*} \in \mathcal{E}$. 


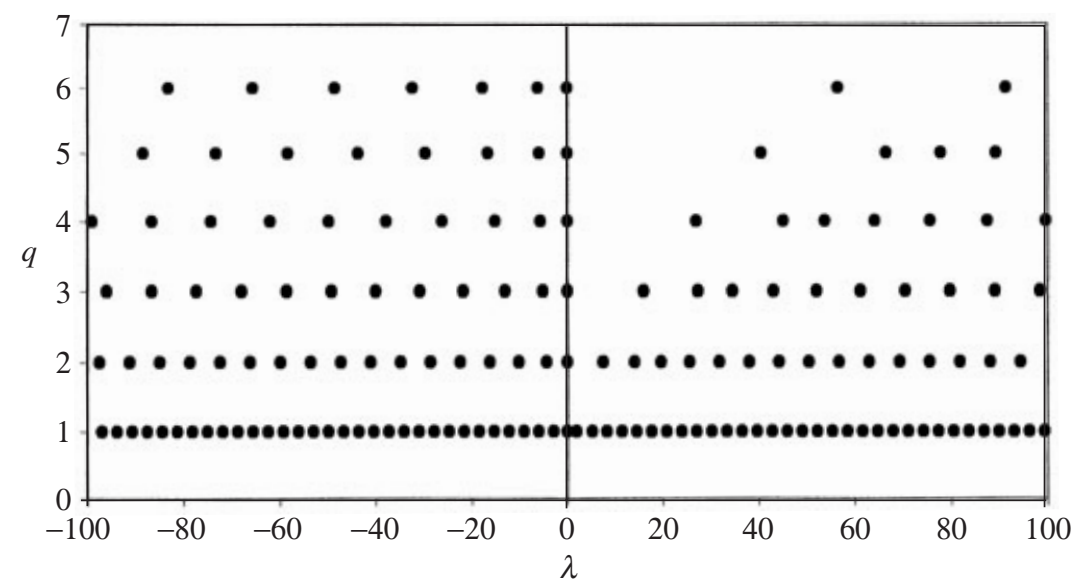

Figure 2. Eigenvalue distribution for $c=1$ and $q=1,2,3,4,5,6$.

$(\Leftarrow)$ Let $\lambda^{*} \in \mathcal{E}$, then, without loss of generality, we have $\lambda^{*}=\lambda_{r}^{+}$for some $r=$ $0,1,2, \ldots$ It follows from Lemma 2.10 that

$$
\left(\lambda^{*}, \lambda^{* 2} / q^{2}\right) \in A \cap\left[\bigcup_{n=0}^{\infty} \Lambda_{n}\right]
$$

and then, via Lemma 2.7, that $\lambda^{*}$ is an eigenvalue of $(\mathrm{E})$.

Remark 2.12. Each eigenvalue $\lambda=\lambda_{r}^{ \pm}(r=0,1,2, \ldots)$ of (E) has a one-dimensional space of eigenfunctions spanned by $\psi=\phi_{r}\left(x, \lambda_{r}^{ \pm}\right):[0,1] \rightarrow \mathbb{R}$. We also note that the eigenfunctions $\phi_{0}\left(x, \lambda_{0}^{ \pm}\right)$both have no zeros for $x \in[0,1]$, while the eigenfunctions $\phi_{r}\left(x, \lambda_{r}^{ \pm}\right)$have exactly $r$ zeros for $x \in[0,1](r=1,2, \ldots)$.

\section{Generalizations}

The approach of the previous section can be applied directly to the following generalization of the eigenvalue problem (E), namely

$$
\begin{gathered}
{\left[x \psi^{\prime}\right]^{\prime}+[H(\lambda)-\lambda G(x)] x \psi=0, \quad x \in(0,1),} \\
\psi(x), \psi^{\prime}(x) \text { bounded as } x \rightarrow 0, \\
\psi^{\prime}(1)=0,
\end{gathered}
$$

which we will denote as $\left(\mathrm{E}^{\prime}\right)$. Here, $G:[0,1] \rightarrow \mathbb{R}$ is now such that $G$ is analytic and strictly positive on $[0,1]$, while $H: \mathbb{C} \rightarrow \mathbb{C}$ is such that $H(\mathbb{R}) \subseteq \mathbb{R}$. As in $\S 3$, we can define functions $\gamma_{n}: \mathbb{R} \rightarrow \mathbb{R}(0,1,2, \ldots)$, which inherit all the properties of the corresponding functions defined in $\S 3$. Following $\S 3$, it is now straightforward to conclude that the set 


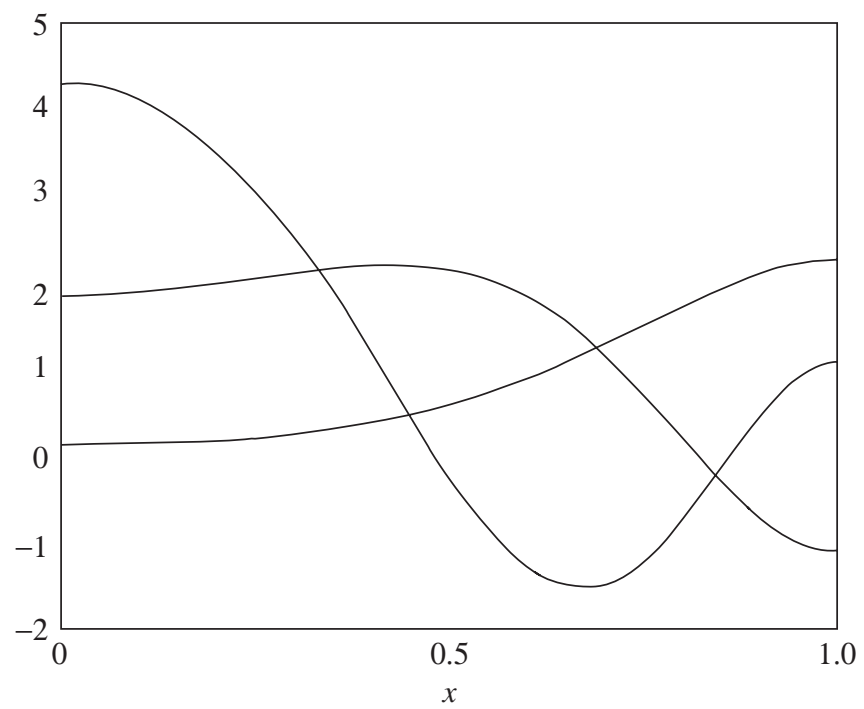

Figure 3. The normalized eigenfunctions associated with eigenvalues $\lambda_{0}^{+}, \lambda_{1}^{+}$and $\lambda_{2}^{+}$when $c=1$ and $q=4$.

of real eigenvalues of $\left(\mathrm{E}^{\prime}\right)$ are precisely those values of $\lambda \in \mathbb{R}$ such that

$$
\lambda \in \bigcup_{n=0}^{\infty} \mathcal{K}_{n},
$$

where

$$
\mathcal{K}_{n}=\left\{\lambda \in \mathbb{R}: \gamma_{n}(\lambda)=H(\lambda)\right\} .
$$

An interesting point to note is that should $H(\lambda) \equiv \gamma_{r}(\lambda)$, for some $r=0,1,2, \ldots$, on $\lambda \in[\alpha, \beta]$, say, then $\lambda^{*}$ is an eigenvalue of $\left(\mathrm{E}^{\prime}\right)$ for each $\lambda^{*} \in[\alpha, \beta]$, and the set of real eigenvalues of $\left(\mathrm{E}^{\prime}\right)$ is no longer discrete. Indeed, if it happens that $H(\lambda) \equiv \gamma_{r}(\lambda)$ for all $\lambda \in \mathbb{R}$ (some $r=0,1,2, \ldots$ ), then $\lambda^{*}$ is an eigenvalue of $\left(\mathrm{E}^{\prime}\right)$ for each $\lambda^{*} \in \mathbb{R}$. In addition, it is clearly possible, for suitable choices of the function $H(\lambda)$, to construct examples where the set $\mathcal{E}^{\prime}$ of real eigenvalues of $\left(\mathrm{E}^{\prime}\right)$ has a number of the following properties:

(i) $\mathcal{E}^{\prime}$ is unbounded above and below;

(ii) $\mathcal{E}^{\prime}$ is bounded below and unbounded above;

(iii) $\mathcal{E}^{\prime}$ is bounded above and unbounded below;

(iv) $\mathcal{E}^{\prime}$ is bounded both above and below;

(v) $\mathcal{E}^{\prime}$ contains an interval of the real line;

(vi) $\mathcal{E}^{\prime}$ is wholly discrete; 
Table 1. Computed eigenvalues $(\lambda)$ for $c=1$ and $q=4,5,6$ in the range $(-100,100)$.

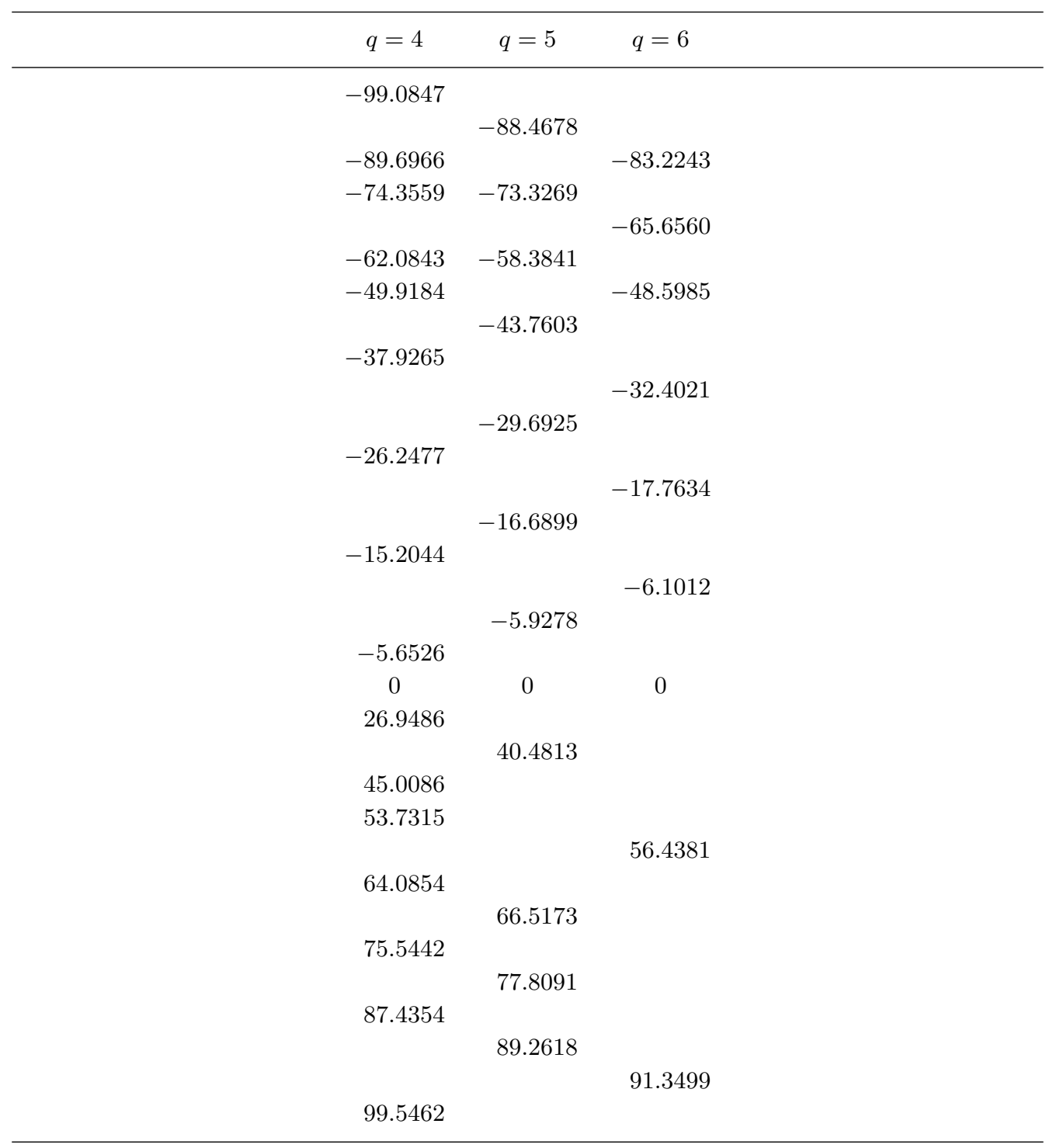

(vii) $\mathcal{E}^{\prime}$ is wholly an interval of the real line;

(viii) $\mathcal{E}^{\prime}$ has an accumulation point.

\section{Numerical method and results}

To numerically find the eigenvalues and eigenfunctions of (E), we first use the invariance of the differential equation under scaling transformations in $\psi$ and the boundedness of 
Table 2. Convergence of ratio of $n$th eigenvalues.

\begin{tabular}{cccccc}
\hline$n$ & 500 & 1000 & 2000 & 3000 & 4000 \\
\hline$\lambda_{n}^{+} / \lambda_{n}^{-}$ & -0.99930 & -0.99964 & -0.99981 & -0.99988 & -0.99991 \\
\hline
\end{tabular}

solution near the origin to turn it into an initial value problem of the form

$$
\left.\begin{array}{c}
\left(x y^{\prime}\right)^{\prime}+\left(\frac{\lambda^{2}}{q^{2}}-\lambda G(x)\right) x y=0, \quad x \in(0,1), \\
y(0)=1, \quad y^{\prime}(0)=0 .
\end{array}\right\}
$$

This can be readily integrated, using the NAG routine D02BBF, for any given $\lambda$ to find $y(1)$ and $y^{\prime}(1)$. An interval search for changes in sign of $y^{\prime}(1)$ will give rough estimates of the positions of the eigenvalues which can be then found more precisely by Newtonian iteration. For any particular eigenvalue the eigenfunction is easily found and a simple quadrature will give $\int_{0}^{1} x y^{2}(x) \mathrm{d} x=a$. The normalized eigenfunction can then be defined as $\tilde{y}=y / \sqrt{a}$. This scheme was efficient and successful in finding the eigenvalues for any positive values of $q$ and $c$. The results of computations for the eigenvalues for $q$ varying and $c$ fixed at unity are shown in Figure 2 and are typical of the results we found. This figure, which should be interpreted qualitatively, demonstrates that as $q$ increases the number of positive eigenvalues within a fixed range, $-100 \leqslant \lambda \leqslant+100$, decreases with the first strictly positive eigenvalues growing in size. There is a slight decrease in the number of negative eigenvalues as $q$ increases but this is not so marked as with the positive ones. There is always an $O(1)$ negative eigenvalue whose size does not vary significantly as $q$ varies. Furthermore, the negative eigenvalues are far more uniformly spaced than the positive ones. This is in line with the inequalities (2.24) and (2.25) when $q \gg 1$.

Table 1 gives precise quantitative information about the size of the eigenvalues for $q=4,5,6$. The numerical results can also be used to confirm the asymptotic results $\lambda_{n}^{ \pm} \sim \pm q \sqrt{\mu_{n}}$ as $n \rightarrow \infty$ to three decimal places. In Table 2 we have displayed $\lambda_{n}^{+} / \lambda_{n}^{-}$ as a function of $n$ when $q=4$, and the approach is accurate to four decimal places for $n=4000$.

Finally, Figure 3 shows the first three normalized eigenfunctions associated with eigenvalues $\lambda_{0}^{+}, \lambda_{1}^{+}$and $\lambda_{2}^{+}$for $c=1, q=4$. It is worth pointing out that the first eigenfunction has no zeros and that the second and third have one and two zeros, respectively, in $[0,1]$, in accordance with Remark 2.12.

\section{References}

1. E. A. Coddington And N. Levinson, Theory of ordinary differential equations (McGraw-Hill, New York, 1955).

2. R. Cooper, J. Billington and A. C. King, Flow and reaction in solid oxide fuel cells, J. Fluid Mech. 411 (2000), 233-262. 
3. R. Copcutt, A. C. King And K. Kendall, Reaction-diffusion of fuel with air between planar solid oxide fuel cells stacked with ceramic felt, Proc. R. Soc. Lond. A 452 (1996), 2639-2653.

4. I. StAKGOLD, Green's functions and boundary value problems (Wiley, New York, 1979). 